\title{
Cuidados de la piel y prevención en dermatitis atópica y alergia a alimentos
}

\author{
Dra. Rosa Elena Huerta Hernández*
}

Aproximadamente un tercio de los pacientes con dermatitis atópica tienen síntomas de alergia a alimentos. ${ }^{1}$

El tratamiento temprano agresivo para disminuir la duración del eccema en niños da como resultado una menor frecuencia de aparición de alergia a alimentos a los dos años de edad. ${ }^{2}$

Más de la mitad de los niños con eccema de inicio temprano de 0-3 meses de edad que ameritaron uso de corticoesteroides tópicos desarrollaron prueba de reto positivo a alimentos a los 12 meses de edad. ${ }^{3}$

En el daño epicutáneo en la dermatitis atópica, participan diversos factores de riesgo, tanto ambientales como genéticos: entre los factores ambientales está la mayor predisposición a infecciones por virus, bacterias y hongos, el uso de detergentes, el uso de emolientes con $\mathrm{pH}$ alcalino, así como la exposición cutánea a alimentos alergénicos a través de una piel dañada, favoreciendo sensibilización y desarrollo de alergia alimentaria. En los factores genéticos están las mutaciones genéticas en las proteínas de la barrera cutánea: filagrina, loricrina y SPINK5 (serine peptidase inhibitor Kazal type 5). ${ }^{4}$

En dos estudios se observó que el tratamiento con emolientes, desde el nacimiento hasta las 32 semanas de vida, podía ayudar en la prevención primaria para dermatitis atópica en niños de alto riesgo con una reducción de hasta el $50 \% .^{5,6}$

Sin embargo, en el estudio BEEP (Barrier Enhancement Eczema Prevention), donde participaron 1,395 niños con historia familiar de atopia, se les aplicó gel doble base y/o crema diprobase desde las tres semanas de nacidos hasta los 12 meses de edad, tres a cuatro veces por semanas con $51 \%$ de apego al tratamiento pero no hubo mejoría en la prevención, retraso o reducción de la gravedad de la dermatitis atópica ni prevención en alergia a alimentos. Además se presentó mayor riesgo de infecciones en piel. ${ }^{7}$

En el estudio PreventADALL se estudiaron 2,701 niños de la población general, donde se les aplicó crema ceridal a un grupo y a otro grupo aceite de baño desde las dos semanas de vida hasta los nueve meses de edad cuatro veces por semana, así como introducción desde los tres meses de edad con cacahuate, leche, huevo y trigo; 8 sin embargo, ninguna de las dos intervenciones produjo una disminución en el desarrollo de dermatitis atópica a los 12 meses de edad, por lo que los autores no sugieren dichas intervenciones como medidas preventivas en dermatitis atópica.

En el estudio PEBBLES (Prevention of Eczema By Barrier Lipid Equilibrium Strategy) se utilizó EpiCeram, que es una crema que contiene ceramida, colesterol, ácidos grasos, con pH 5 con una composición similar al vérnix caseoso. Se ha reportado que el EpiCeram puede restaurar la barrera a más del 90\% cuatro horas después de su aplicación en pacientes con dermatitis atópica, y tiene efectos similares a los corticoesteroides de mediana potencia. Este producto se aplicó a recién nacidos con antecedentes heredofamiliares de atopia hasta los seis meses de edad dos veces al día en un total de 80 pacientes. Los autores observaron una disminución en la aparición de dermatitis atópica y disminución en la sensibilización a los alimentos a los 12 meses de edad. ${ }^{9}$ Este mismo estudio se está realizando en un mayor número de recién nacidos de alto riesgo (760 niños) pero aún están pendientes los resultados. ${ }^{10}$

En otra investigación se estudiaron 45 niños de tres meses a siete años de edad con dermatitis atópica y/o piel seca y se aplicó Aveeno, loción lubricante, y EpiCeram crema y se observó una mejor respuesta en cuanto a la disminución de la TEWL (pérdida de agua transepidérmica) con el uso de EpiCeram comparada con Aveeno. La loción de Aveeno contiene una base de petrolato más parafina con dimeticona al $1.2 \%$.

La crema EpiCeram contiene tres lípidos esenciales: ceramidas, ácido linoleico conjugado y colesterol con $\mathrm{pH}$ de $5 .^{11}$

* Alergólogo Pediatra. Clínica de Alergia Pediátrica. Pachuca, Hidalgo. 
Vol. 29, Núm. 2 • Mayo-Agosto 2020

El mismo grupo de trabajo ahora estudió a 16 niños con dermatitis atópica, e igual se le aplicó a un grupo EpiCeram y a otro grupo Aveeno, después de 12 semanas de aplicación midieron perfil de citocinas. En el grupo EpiCeram observaron disminución en los niveles de IgE total, incremento en IgG4 e incremento en IL-10 y disminución en IL-4. ${ }^{12}$

Por otro lado se ha observado que la participación del Staphylococcus aureus juega un papel importante en la dermatitis atópica y en la alergia a alimentos, ya que la presencia de este microorganismo favorece el aumento de IgE específica a alimentos como huevo y cacahuate, inhibe el desarrollo de tolerancia oral al cacahuate, incrementa la gravedad del eccema y retarda la resolución de la alergia al huevo. ${ }^{13}$

En otro trabajo, el tratamiento temprano agresivo y proactivo tópico con corticoesteroides para disminuir la duración del eccema ha resultado en la disminución de la alergia a alimentos a los dos años de edad. ${ }^{2}$

Finalmente, las perspectivas futuras para la prevención primaria y secundaria, tanto en dermatitis atópica como en alergia a alimentos, dependerán de identificar el inicio óptimo y duración de las intervenciones preventivas mencionadas, favorecer mayor grado de adherencia en los tratamientos, conocer mejor las características de los diferentes emolientes y valorar el papel que desempeñan los siguientes factores: la exposición a alimentos en el medio ambiente, presencia del Staphylococcus aureus y uso de agentes antiinflamatorios.

\section{BIBLIOGRAFÍA}

1. Gupta R, Warren CM, Smith BM et al. Prevalence and severity of food allergy among US adults. JAMA Netw Open. 2019; 2: e185630.

2. Miyaji Y, Yang L, Yamamoto-Hanada K, Narita M, Saito $H$, Ohya $Y$. Earlier aggressive treatment to shorten the duration of eczema in infants resulted in fewer food allergies at 2 years of age. J Allergy Clin Immunol Pract. 2020; 8 (5): 17211724.e6.

3. Allen KJ, Koplin JJ. Prospect for prevention in food allergy. $J$ Allergy Clin Immunol Pract. 2016; 4 (2): 215-220.
4. Brough HA, Nadeau KC, Sindher SB et al. Epicutaneous sensitization in the development of food allergy: What is the evidence and how can this be prevented? Allergy. 2020; 10.1111/all.14304.

5. Simpson EL. Emollient enhancement of the skin barrier from birth offers effective atopic dermatitis prevention. J Allergy Clin Immunol. 2014; 134: 818-823.

6. Horimukay K. Application of moisturizer to neonates prevents development of atopic dermatitis. J Allergy Clin Immunol. 2014; 134: 824-830.

7. Chalmers JR, Haines $\mathrm{RH}$, Bradshaw LE et al. Daily emollient during infancy for prevention of eczema: the BEEP randomised controlled trial. Lancet. 2020; 395 (10228): 962972.

8. Skjerven HO, Rehbinder EM, Vettukattil $\mathrm{R}$ et al. Skin emollient and early complementary feeding to prevent infant atopic dermatitis (PreventADALL): a factorial, multicentre, cluster-randomised trial. Lancet. 2020; 395 (10228): 951-961.

9. Lowe AJ, Su JC, Allen $\mathrm{KJ}$ et al. A randomized trial of a barrier lipid replacement strategy for the prevention of atopic dermatitis and allergic sensitization: the PEBBLES pilot study. Br J Dermatol. 2018; 178 (1): e19-e21.

10. Lowe A, Su J, Tang M et al. PEBBLES study protocol: a randomised controlled trial to prevent atopic dermatitis, food allergy and sensitisation in infants with a family history of allergic disease using a skin barrier improvement strategy. BMJ Open. 2019; 9 (3): e024594.

11. Sindher S, Alkotob SS, Shojinaga MN et al. Pilot study measuring transepidermal water loss (TEWL) in children suggests trilipid cream is more effective than a paraffin-based emollient. Allergy. 2020; 10.1111/all.14275.

12. Sindher S, Alkotob SS, Shojinaga MN, Hamilton R, Chan $S$, Cao $S$ et al. Increases in plasma IgG4/lgE with trilipid vs paraffin/petrolatum-based emollients for dry skin/eczema. Pediatr Allergy Immunol. 2020.

13. Tsilochristou $\mathrm{O}$, du Toit G, Sayre PH et al. Association of Staphylococcus aureus colonization with food allergy occurs independently of eczema severity. J Allergy Clin Immunol. 2019; 144 (2): 494-503.

Dirección para correspondencia: Dra. Rosa Elena Huerta Hernández Articulo 27 Núm. 102, Col. Fraccionamiento Constitución, 42080, Pachuca de Soto, Hidalgo. E-mail: rehhmexico@gmail.com 